SISTEMA
ELETRÔNICO
DE REVISTAS
SER I UFPR

\title{
La geoecología de los paisajes como base teórico-metodológica para incorporar la dimensión tecnológica a la temática ambiental
}

\section{A geoecologia das paisagens como base teórico-metodológica para incorporar a dimensão tecnológica à temática ambiental}

\section{Geoecology of landscapes as theoretical-methodological basis to incorporate technological dimension in environmental thematic}

\author{
José Manuel MATEO RODRIGUES ${ }^{1 *}$, Edson Vicente SILVA², Adriano Severo FIGUEIRÓ ${ }^{3}$ \\ ${ }^{1}$ Universidad de la Habana, Habana, Cuba. \\ ${ }^{2}$ Universidade Federal do Ceará (UFC), Fortaleza, CE, Brasil. \\ ${ }^{3}$ Universidade Federal de Santa Maria (UFSM), Santa Maria, RS, Brasil. \\ *E-mail de contacto: mateopaisajescuba@gmail.com
}

Artículo recibido el 12 de marzo, 2019, versión final aceptada el 20 de mayo, 2019.

RESUMEN: En el artículo se pretende discutir cuatro cuestiones básicas: a) la esencia de la dimensión tecnológica de la cuestión ambiental; b) la noción de medio ambiente; c) la necesidad de la Geoecología de los Paisajes como fundamento teórico-metodológico de la cuestión ambiental; d) la posibilidad de utilizar la Geoecología de los Paisajes en las herramientas aplicadas de la planificación y la gestión ambiental, y en los mecanismos innovadores de la Geo tecnología. De tal manera se promueve la Geoecología de los Paisajes, porque puede constituir la base teórico-metodológica que sustente la construcción de la dimensión tecnológica en la incorporación de la dimensión ambiental a los procesos de desarrollo.

Palavras claves: ambiente; técnica; herramienta; desarrollo; planificación.

RESUMO: O artigo objetiva discutir quatro questões básicas: a) a essência da dimensão tecnológica da questão ambiental; b) a noção de meio ambiente; c) a necessidade da Geoecologia das Paisagens como fundamentação teórico-metodológica da questão ambiental; d) a possibilidade de utilizar a Geoecologia das Paisagens nas ferramentas aplicadas de planejamento e gestão ambiental, e nos mecanismos inovadores da tecnologia Geo. Desse modo, 
promove-se a Geoecologia das Paisagens, pois ela pode constituir a base teórico-metodológica que sustenta a construção da dimensão tecnológica na incorporação da dimensão ambiental aos processos de desenvolvimento.

Palavras-chave: ambiente; técnica; ferramenta; desenvolvimento; planejamento.

ABSTRACT: The article aims to discuss four basic issues: a) the essence of the technological dimension of the environmental issue; b) the notion of the environment; c) the need for the Geoecology of Landscapes as a theoreticalmethodological foundation of the environmental issue; d) the possibility of using the Geoecology of Landscapes in the applied tools of environmental planning and management, and in the innovative mechanisms of Geo technology. In this way, the Geoecology of Landscapes is promoted, because it can constitute the theoreticalmethodological basis that sustains the construction of the technological dimension in the incorporation of the environmental dimension to the development processes.

Keywords: environment; technique; tool; development; planning.

\section{La noción de medio ambiente}

Cada vez más se percibe la imperiosa necesidad de garantizar una utilización de la técnica y la tecnología de forma que se articule de manera racional con el medio ambiente en el cual se incorpora. El desarrollo sostenible ha considerado una dimensión tecnológica para su conquista y construcción, sin embargo, la introducción de la tecnología en el contexto ambiental exige de una visión paradigmática que tal visión sea coherente en la formulación de una relación armónica entre los sistemas humanos, los ambientales y los tecnológicos. La generalidad de los paradigmas utilizados enfatiza en un aspecto de la relación naturaleza-sociedad, mientras la concepción de la Geoecologia de los Paisajes, formulada desde mediados del siglo XIX por Alejandro de Humboldt y V. V. Dokuchaev, se caracteriza por una visión integradora y totalizadora desde una perspectiva dialéctica a la hora de entender al medio geográfico de la sociedad humana.

La cuestión ambiental ha irrumpido en la ciencia de manera impetuosa a partir de los años 60 del siglo XX. Su formulación ha estado fuertemente matizada por el protagonismo disciplinario de la Ecología desde una posición biologizante. La Geografía, que había sido la pionera de este campo del saber, no logró colocar en protagonismo todo su instrumental teórico y metodológico.

Sin embargo, la cuestión ambiental enfrenta retos mayores que obligan a todas las ciencias y la filosofía a revisar todo el instrumental científico creado, lo que explica la necesidad de valorar el papel de la Geografía, desde una visión de la teoría del conocimiento en la problemática ambiental. En este sentido, se rescata el papel de la Geoecología de los Paisajes como enfoque nacido de la Geografía, que tiene como propósito, desde una mirada holística, concreta y multiescalar, visualizar el funcionamiento ambiental de las relaciones de los seres humanos con su medio natural y social. De tal manera el objetivo del presente artículo es argumentar el papel de la Geoecología como un enfoque capaz de convertirse en el fundamento científico-metodológico del paradigma ambiental. En este sentido, se asumen las siguientes definiciones de medio ambiente abajo.

Medio geográfico de la sociedad: es el espacio de la superficie del planeta Tierra en el cual vive y se desarrolla la humanidad, incluyendo el medio natural y el social. Es la propia esfera geográfica, cuya evolución la ha conducido al período geológico antropocénico y que ha condicionado el surgimiento del Hombre, la formación de las 
sociedades humanas y el desarrollo ininterrumpido de las interconexiones entre los seres humanos con el medio, dando lugar a la formación del medio ambiente humano (Timashev, 1999).

Medio ambiente humano: es el medio antropocéntrico y antropoecológico. Es el conjunto de condiciones naturales (abióticas y bióticas), antropo-naturales, tecno-génicas y sociales, en las cuales vive y vivirá la humanidad, siendo el resultado de su profunda transformación que ha incluido la incorporación en la misma de objetos tecno-génicos (Timashev, 1999). Hace parte del medio ambiente de la sociedad y focaliza su atención, en los seres humanos como centro del sistema.

A partir de 1990 con el proceso de "hibridación" del conocimiento geográfico se ha desarrollado una visión sistémica del Medio Ambiente en que tratan de integrarse los conceptos desarrollados por la Ecología Humana y la Geografía. Para ello se ha tomado como referencia, toda la herencia epistemológica en la formulación del concepto y su interpretación desde la visión espacial y los conceptos de medio geográfico desarrollada por la Geografía (Santos, 1994; 1996; Santos \& Silveira,
2002; Rodriguez \& da Silva, 2006; Baklanov, 2010; Rodriguez, 2015; Diakonov et al., 2014).

Esta visión opera con conceptos, que establecen los elementos que interactúan entre sí, para formar una totalidad sistémica para definir el medio ambiente (Gallopin, 1980): i) Sistema humano: el ser humano como centro del medio ambiente, sus características físicas y demográficas, comportamientos, percepciones, conductas; ii) Medio natural: componentes y complejos naturales, que forman la base natural del Medio ambiente; iii) Medio social: entorno económico, social, cultural y sus componentes y complejos.

Según tal definición, el medio ambiente forma un sistema condicionado por la interacción del medio natural y el social, con el ser humano, teniendo a éste como eje central de funcionamiento y de estructuración, en una visión de relaciones objeto/ sujeto. Por lo tanto, el medio ambiente en esta visión no está inserto completamente en la naturaleza, sino que es el resultado de la interacción de dos formaciones materiales relativamente autónomas, aunque interdependientes: Naturaleza y Sociedad (Figura 1).

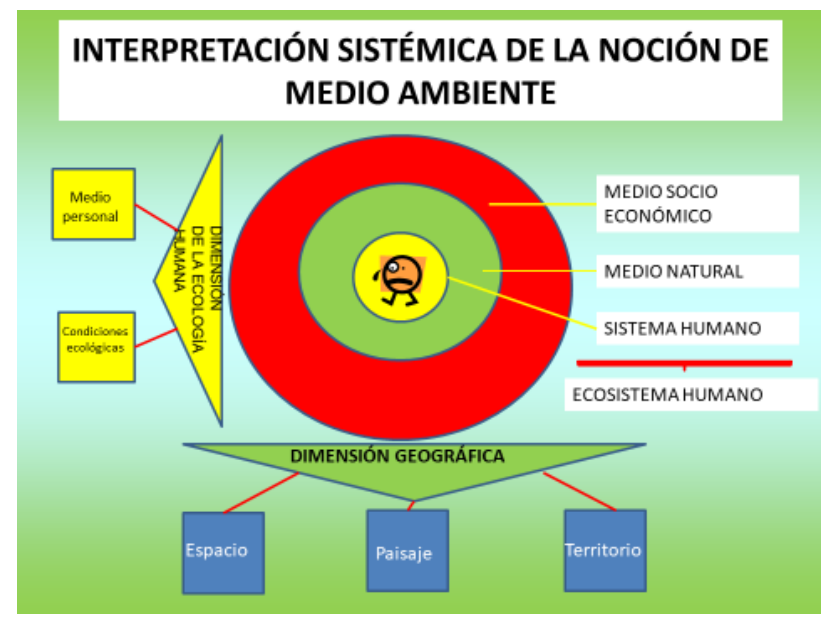

FIGURA 1 - Interpretación sistémica de la noción de medio ambiente. FUENTE: Gallopin (1980); Rodriguez (2015). 
En resumen, cada una de las grandes matrices epistemológicas ha brindado su aporte y enfatizan en un u otro aspecto de la cuestión ambiental. A partir del siglo XXI lo que se observa es un proceso de hibridación de las ciencias, tratándose de articular métodos, técnicas y procedimientos desarrollados por diferentes marcos teóricos, pero partiendo de un modelo epistémico dado. Esto es lo que predomina en la actualidad, utilizando en gran parte a la visión dialéctica como el centro o corazón de las articulaciones de las diferentes teorías (Rodriguez, 2015).

La Geoecología de los Paisajes, propone una visión ampliada del medio ambiente, en la que el sistema humano, interactúa con el paisaje como sistema de conceptos (paisaje natural/antroponatural y cultural) (Nikolaiev, 2006; Kalutskov, 2008; Rodriguez, 2015).

\section{La dimensión tecnológica en el análisis ambiental}

El discurso sobre el papel que juegan la ciencia y la tecnología respecto al desarrollo social, ha pasado por diferentes etapas que van desde un optimismo exagerado hasta su rechazo total. A partir del Renacimiento se desarrolla una imagen de la ciencia, que la caracteriza en lo fundamental desde el siglo XVII hasta mediados del siglo XX, vinculada al progreso social. Pero es, en realidad, a partir de la segunda mitad del siglo XX que se puede hablar de una actitud de temor, cuestionamiento y rechazo a la tecnología, actitud vinculada a los actos de protesta de los movimientos ambientalistas en el capitalismo de los años 60, a los diferentes tipos de desastres ocasionados al medio ambiente, a la psicología del hombre, a los problemas que surgen con la globalización de la economía y la cultura, al desarrollo de la biotecnología y al desarrollo de los medios de comunicación masiva (Rodriguez \& Suárez, 2000).

Ya en la Agenda 21 de las Naciones Unidas, documento programático elaborado en la Cumbre de Río, aparece una lista de problemas que abarca todos los elementos constituyentes del Planeta y todas las actividades de la sociedad. La crisis ambiental, concebida como un fenómeno cualitativamente diferente, se debe a que los lazos eco sistémicos globales están amenazados, porque los sistemas positivos de retroalimentación impuestos por las tendencias de nuestra dinámica civilizadora, no son ya compensados por los recursos negativos de retroalimentación de la naturaleza (Sosa, 1995). La crisis ambiental es, por lo tanto, una crisis de civilización.

Del delicado estado ambiental del planeta Tierra no se puede culpar sólo a la tecnología, ya que no actúa en un vacío político-ideológico. La técnica es definida, por Ortega \& Gasset (2004), como la forma en que el hombre impone su comportamiento a la Naturaleza, para la satisfacción de sus necesidades; es el intermediario mediante el cual el hombre la utiliza, modifica y transforma, y se encuentra en estrecho vínculo con los diferentes componentes del sistema cultural (el sistema biofísico natural, la organización social y hasta los símbolos e ideas que constituyen el imaginario colectivo). ¿Cómo ha coadyuvado la tecnología al proceso de deterioro y degradación ambiental? Una primera respuesta, estaría dirigida a explicar las "anomalías", con las cuales la tecnología degrada al medio natural y en este sentido podrían definirse tres posibilidades:

a) Cuando la tecnología es ecológicamente ineficiente, conduciendo al surgimiento de procesos degradantes de todo tipo: son las tecnologías energéticamente deficientes, generadoras de residuos, destructoras de sistemas ambientales;

b) Cuando se instalan dispositivos técnicos que no corresponden a la estructura y el funcionamiento 
de los sistemas ambientales, así ocurre con los sistemas técnicos gigantes, el llamado "gigantismo", con las estructuras tecnológicas que tampoco se ajustan a las estructuras del eco o geosistemas, todo lo cual conduce a procesos de degradación ambiental y productiva;

c) Cuando el manejo de los sistemas técnicos es incorrecto o desarticulado, dando lugar a procesos de degradación. Por ejemplo, las normas de introducción de energía y de sustancias que no pueden ser absorbidas por los sistemas naturales, $\mathrm{o}$ aquellos sistemas de explotación que no permiten la regeneración de los recursos.

Una segunda respuesta, está dirigida a entender con mucha mayor amplitud, la interacción Naturaleza-Sociedad, mediante la interferencia tecnológica. En este sentido, es interesante analizar la cuestión ambiental a nivel global, siendo numerosos los intentos de comprender el estado actual y pronosticar el futuro ambiental del planeta Tierra; el pronóstico presagia que para mediados del siglo XXI se producirá un estado ambiental catastrófico a nivel mundial, el cual pudiera conducir a una crisis generalizada a nivel social. Dicho estado catastrófico se manifestará por un descenso significativo de recursos, un aumento de la degradación ambiental y una disminución de la producción de alimentos.

El resultado sería que, para mediados del siglo XXI, se podría comenzar a revertir la situación de crisis ambiental, actualmente prevaleciente y pasar a una nueva fase de crecimiento y prosperidad. Para encontrar la respuesta al estado actual de la crisis ambiental se utilizan dos enfoques:

Para el enfoque tecnicista, la tecnología ha sido la responsable del crítico estado ambiental de la Tierra. El proyecto del Iluminismo del siglo XVIII se basaba en la creencia de que los seres humanos podrían crear una sociedad armónicamente articulada con el empleo de la razón en los asuntos humanos, que la Naturaleza podía transformarse en la fuente de la abundancia material mediante la aplicación de la ciencia y la tecnología, mientras que la aplicación de la razón a la sociedad humana, conduciría a la felicidad. Según esta posición, la solución requeriría el cambio de las tecnologías; o sea la introducción de aquellas que tuvieran en cuenta la limitación espacial del planeta y la limitación de los recursos. La emergencia de una razón técnica, conduciría a una legitimización integralmente auto-centrada, situando a la técnica como algo superior a los conflictos y a las disputas.

Para el enfoque socio político, el núcleo de la actual crisis ambiental está en la esencia antiecológica del capitalismo y de su modo de producción, como gran destructor de la Naturaleza. La lógica interna de crecimiento o muerte del sistema capitalista, está en contradicción dialéctica, ya que el capital debe crecer sin límites, mientras que la Tierra tiene límites. Se parte, así, del hecho de que la tecnología está instituida en un campo de relaciones intersubjetivas, lo cual permite afirmar que la ciencia y la tecnología no son neutras. La ciencia y la tecnología son fenómenos sociales, constituyen variables dependientes respecto al conjunto de las relaciones socio-económicas que existen y se desarrollan en una sociedad determinada.

Se concluye, así, que la tecnología es una condición necesaria pero no suficiente para resolver los problemas que enfrenta la humanidad. La solución de los problemas ambientales no sería sólo de naturaleza tecnológica, sino de una opción político-cultural a la que la tecnología deberá estar subordinada. Se parte de que al capitalismo le ha sido inherente un sistema expansivo, basado en dos pilares: el consumismo, o sea la ideología de que la felicidad humana depende absoluta y exclusivamente del consumo de bienes y servicios, de mercadurías; y la movilización de la investigación 
científica y la innovación tecnológica en servicios de promoción de bienes, y de mercancías (Benton et al., 1995).

La tecnología es fundamental en la interrelación entre la Naturaleza y la Sociedad, al establecer los flujos de energía, materia e información necesarios para la supervivencia y el funcionamiento de los sistemas naturales y sociales. De tal manera la dimensión tecnológica del medio ambiente y del Desarrollo Sostenible deberá cumplir con los siguientes requisitos:

La creación e instrumentación de un conjunto de tecnologías orientadas hacia la sustentabilidad (sostenibles, apropiadas, alternativas, etc.), que impliquen al mismo tiempo una desmaterialización de los procesos productivos y sociales, la eficacia y productividad máxima de energía y materias primas, limitada producción de emisiones y la compatibilidad con los procesos naturales. La incorporación del concepto de eficiencia ecológica o ecoeficiencia, concebida como el logro de productos y servicios a precios competitivos que satisfagan las necesidades humanas y brinden calidad de vida, al mismo tiempo que reduzcan de forma progresiva el impacto ambiental y la intensidad de uso de los recursos mediante la reducción del ciclo de vida a un nivel si no el mínimo, al menos menor que la capacidad de carga de la Tierra (Fussler, 1995). Para hacer efectivo este concepto se debe tomar como elementos definitivos las pautas que están en la base de los procesos biofísicos de los sistemas ambientales. Las dimensiones o elementos del sistema de la ecoeficiencia incluyen, la masa, energía, calidad ambiental, salud humana, reciclabilidad, naturalidad, renovabilidad, funcionabilidad y la durabilidad.

La combinación de técnicas tradicionales y modernas de diferentes escalas, técnicas suaves y de escala reducida, tecnologías intermedias dependientes de la oferta económica, diferentes procesos tecnológicos adecuados para la transformación de cada recurso particular, ello implicaría adoptar el pluralismo, la hibridización y la adaptación tecnológica a situaciones locales y contextos regionales para crear condiciones de sustentabilidad.

La utilización ambiental del espacio de acuerdo a la vocación del potencial y aptitud de cada sistema ambiental territorial para lograr la formación de estructuras espaciales estables podría lograrse con la implantación de procedimientos de planificación y gestión ambiental y territorial de manera articulada.

El fortalecimiento de la capacidad de generación endógena, creación y transferencia de tecnologías; el acceso, la asequibilidad de la información científico-tecnológica, de patentes y de la propiedad intelectual, y la formación de una masa crítica de capacidad de investigación - desarrollo (I $+\mathrm{D})$ para la difusión, utilización y creación de tecnologías orientadas hacia la sustentabilidad.

En síntesis, todo lo anterior requiere la implementación de cierto nivel de productividad eco-tecnológica, interdependiente de los procesos culturales y ecológicos, que implica la construcción de una tecnoestructura normada por un conjunto de valores sociales y condiciones naturales, que se promueva la conservación de ciertas estructuras básicas de los sistemas ambientales, que soporten la estabilidad del potencial productivo a largo plazo y la capacidad de regeneración de los recursos (Leff, 1994). Ello obligaría al desarrollo de tecnologías dirigidas a preservar y fortalecer la integridad cultural de las comunidades, que requieran poco capital para su construcción y puesta en marcha. Ofrecerán un alto nivel de empleo, ya que no exigen una capacitación especializada para poder manejarlas, favorecerán la gestión comunitaria y solidaria del proceso de trabajo, a la vez que serán adaptables social y ambientalmente a localidades específicas. 
Se caracterizan, además, por ejercer un impacto ambiental mínimo, que favorece el funcionamiento estable de los sistemas ambientales, basándose en recursos renovables y materiales locales, distribuyéndose de manera dispersa en el territorio y no concentrada, y que emiten desechos reabsorbibles y reutilizables. En cuanto a las tecnologías orientadas a la sustentabilidad se establecen conceptos que manifiestan algunas diferencias en cuanto a la esencia de las definiciones empleadas. Así:

Las sostenibles ponen la mayor atención en la conservación de la capacidad de carga de los sistemas ambientales, a la disponibilidad de los recursos y la integridad cultural (Thayer, 1994).

Las apropiadas o adecuadas son aquellas que al introducirse en una sociedad crean un proceso de auto reforzamiento interno de la propia comunidad, entendiendo esta en términos de las personas que la forman, las actividades que realizan y el entorno en que se encuentran.

Las ecológicamente racionales, son aquellas que generan pocos residuos y en consecuencia tienen un bajo efecto contaminante. Se incluyen las que se denominan "de etapa final" para el tratamiento de la contaminación cuando esta ya se ha producido.

Ninguna tecnología o sistema de manejo opera en el vacío, ellas son apropiadas sólo bajo ciertas condiciones ambientales, socio culturales, e incluso institucionales. Ellas serán aquellas que mantengan e incrementen las reservas de recursos naturales renovables, promuevan la sustitución de recursos no renovables por recursos renovables. Al mismo tiempo, deben satisfacer las diversas expectativas de los pobladores, como asegurar la auto subsistencia, aumentar el ingreso y disminuir los riesgos. La sustentabilidad no es, como puede apreciarse, una condición mágica que sucede porque sí y carece de costos, se da, cuando existen incentivos para que ocurra. Se trata de entender qué condiciones son necesarias para mantener e implementar tecnologías o sistemas de manejo sustentables, o cuáles habría que cambiar para transformar lo no sostenible en uno sustentable (Bellón, 1997).

El mundo se vuelve cada día más complicado, y reclama de cada individuo estar en condiciones de hacer frente a esta complejidad. La innovación científico-tecnológica, no puede ya analizarse como un proceso lineal y aislado, donde predomine sólo una lógica autónoma. Exige ser analizada en un contexto donde los aspectos sociales y naturales se vean de forma dialéctica, es decir necesita ser enfocada "natural y socio ambientalmente". La Geoecología de los paisajes en este sentido, puede desempeñar el papel de instrumento teórico-metodológico para incorporar la dimensión tecnológica al proceso de construcción y producción del medio ambiente.

\section{La Geoecología de los paisajes como una dirección del pensamiento ambientalista}

De todas las corrientes que se han ido desarrollando en casi 200 años de existencia de la Geografía, la Geoecología de los Paisajes se erige como la corriente académica más potente y fundamentada. Ella es heredera del paisajismo, corriente desarrollada en los años 20 del siglo XX, basada más que todo en un materialismo espontáneo, como la única alternativa científica en plena efervescencia de la Geografía Moderna, en la que dominaban corrientes positivistas, deterministas e historicistas. La Geoecología como dirección científica inter y transdisciplinaria tiene como objetivo la investigación de la ecosfera como sistema supercomplejo, la esfera de interacción del geo esferas y la humanidad (Golubev, 2008). Metodológicamente se basa en las ciencias naturales de la Tierra. 
Si bien la elaboración de la concepción de paisajes, tuvo que ver con ciertos períodos en que predominaba un determinado paradigma, al mismo tiempo de forma paralela se llevaba a cabo una sobrexposición e interpenetración de diferentes visiones, partiendo de esas premisas es que pueden establecerse las siguientes etapas en la formulación del concepto de paisaje y la estructuración de la corriente paisajística (Rodriguez et al., 2004):

Formulación inicial, correspondiente a finales del siglo XIX.

La etapa antropohumana de la primera mitad del siglo XX:

La etapa racionalista-naturalista, de la primera mitad del siglo XX.

La etapa geosistémica, de los años 1950-1980.

La etapa cultural, de 1990-2000

La etapa de hibridación del siglo XXI

En la formulación del concepto de paisajes, Alexander von Humboldt tuvo un papel fundador, lo definió de manera concisa como el carácter total de una región de la Tierra (Humboldt, 1814). Además de concebir al paisaje como una totalidad natural, lo visualizó desde las dimensiones humanas y culturales, e incluso estéticas y como la gente lo percibe desde los aparatos mentales (Zonnelveld, 1995). Humboldt logró romper con la visión mecanicista de considerar a la naturaleza como un conjunto amórfico de componentes y elementos, por el contrario, consideró a la Naturaleza como el reflejo y la interacción holística de fenómenos (Wulf, 2016).

Dokuchaev en Rusia, casi simultáneamente, también desarrolló el concepto de paisaje (landsha$\mathrm{ft}$ ). En este sentido su aporte estuvo vinculado a tres cuestiones fundamentales: considerar al suelo como un componente esencial, y visualizarlo como el "espejo de los paisajes"; a establecer la ley de la zonalidad geográfica latitudinal como la regularidad fundamental en la diferenciación de los paisajes y de todos los componentes y elementos naturales, e incluso humanos, y a considerar por primera vez la noción de cambio de los paisajes, como la transformación ambiental, que incluía al cambio climático. Ya a fines del siglo XIX, Dokuchaev, en 1948, demostró de forma clásica el uso no solo del enfoque paisajístico sino el verdadero enfoque ecólogo-paisajístico al analizar el uso de la naturaleza, teniendo en cuenta constantemente al hombre y a la sociedad. Al estudiar el problema de la lucha con la seca que determinaba cosechas críticas y hambres en la faja de las tierras no negras de Rusia, le dio un papel exclusivo a todo el complejo del paisaje para la existencia del hombre, y para las acciones productivas (Timashev, 2008).

La etapa antropohumana de la primera mitad del siglo XX:

En Alemania se consolidó la tradición humanista y sociológica del landshaft (paisaje), que en gran parte heredó las tradiciones de la antropo geografía alemana de Ratzel, de principios de siglo XX, y de Hettner, de los años 1930. Ha sido tradicional la relación de la ciencia del landshaft alemán con la filosofía. Es sintomático que Immanuel Kant fue profesor de geografía y que la clasificación de la ciencia en esencial, cronológica y cromática de manera fundamental movimentó el desarrollo de la propia Geografía y la consolidación del enfoque corológico de Schluter, propuesto em los años 1920. Él lo interpretó de manera compleja como la unidad de los objetos naturales, culturales, accesibles a la percepción del hombre, para ello le prestó significativa atención al papel del hombre social, a la comunidad territorial de las personas en la formación del propio landshaft cultural. Al mismo tiempo diferentes autores alemanes (Schluter, Passarge, Richthoffen), van instrumentando con posterioridad la noción de paisaje natural, comenzándose a 
elaborar todo un sistema de unidades taxonómicas. La escuela alemana entendió los paisajes como plasmaciones morfológicas del territorio y lo vio en dos direcciones: el paisaje natural y el cultural (Martínez de Pisón, 1999).

En esta época se desarrolla la noción de paisaje geográfico según Carl Ortwin Sauer, que es el resultado de la acción de la cultura, a lo largo del tiempo, sobre el paisaje natural (Sauer, 1925). Sauer hereda la concepción original morfológica de Humboldt, pero introduce la acción humana de los agentes culturales, para él, la cultura es el agente, el área natural es el medio y el paisaje cultural es el resultado. De manera magistral, logra articular en la Geografía de los Paisajes a la Geografía Física, la Geografía Histórica y la Geografía Cultural, considerando a la Geografía Humana “. . . la cual se ocupa únicamente de instituciones humanas o culturas, y puede ser definida como el problema de la "Standorf" o localización de maneras de vivir..." (Sauer, 1925, p. 24) “...y de la diferenciación de áreas de las actividades humanas..." (p. 25). Considera así a la Geografía Humana como a la propia Geografía teniendo como objetivo "... el estudio comparativo de culturas localizadas en áreas... consideradas como paisaje cultural" (p. 27). Sin embargo, a pesar de considerar a la naturaleza como el punto de partida para la formación del landshaft (paisaje) cultural, en la concepción de Sauer, la naturaleza más que todo era un fondo no suficiente dibujado, caracterizado y sistematizado, privilegiando la acción humana, en una especie de determinismo socio cultural.

Por esta causa el problema de la duplicidad del landshaft se analizó como terminológica-conceptual, o sea, el problema de la duplicidad del concepto de landshaft. En la Geografía anglo sajona se formó una peculiaridad fundamental de la Geografía de los landshafts que fue el predominio de lo sociológico sobre lo étnico en las investigaciones del landshaft cultural americano, cuyo representante es Harsthorne, en el paso a la década de 1940. Este momento (la influencia de la bien desarrollada sociología americana) se manifiesta en la terminología a ser utilizada en el paisaje (landshaft) cultural, utilizándose frecuentemente el término "grupo social" y no "comunidad étnica". Siendo este, uno de los problemas fundamentales para la crítica.

En Francia, la problemática de los landshaft (más exactamente del paisaje) se consideró siempre en el contexto cultural. Tal enfoque fue asumido y desarrollado en torno al geógrafo e historiador francés Paul Vidal de la Blache en los años vinte del siglo XX, para el cual la noción de género de vida, era el concepto fundamentalmente que relacionaba en un todo a la cultura y al landshaft en la corriente posibilista. Para esta visión, el paisaje es la expresión del género de vida, o sea de los artefactos creados por los seres humanos y del estilo de vida, desde una posición y un punto de partida antropo y humano céntrico. En Francia adquirió notable entidad el concepto de paisaje como plasmación tangible espacial, como "rugosidad" o "artefacto" geográfico producido por la adaptación del hombre al medio y del medio al hombre como expresión del género de vida agrario y urbano, como decantación de la relación entre la civilización y su espacio (Martínez de Pisón, 1999).

En Rusia, entre los más importantes antropogeógrafos de la primera parte del siglo XX se distinguen los nombres de D. N. Anuchin; V. P. Semeniov-Tian Shan; A. A. Kruber; P. N. Saviistskii; L. S. Berg. Sin dudas, aquí está presente la decisiva influencia de la geografía alemana de corte sociológico - cultural. En Rusia, entre 1910- 1930 se desarrolló una concepción de paisaje de corte humanista y cultural, aunque partiendo del natural como punto de partida para entender la superficie del globo terráqueo, conforme a la tradición natu- 
ralista rusa se designó como Antropogeográfica al capítulo de la Geografía que consideraba a la Tierra como el lugar del hábitat del hombre. El primero de los geógrafos rusos que utilizó el término landshaft cultural fue Berg en 1915, e introdujo la idea acerca de los dos objetos del landshaft: el landshaft natural y el landshaft cultural. La Antropogeográfica de aquellos años estaba fuertemente relacionada con las ciencias humanitarias: la historia, etnografía, toponimia y el foklor.

Por otra parte, bajo la influencia de la Geografía, si bien, en este período se va consolidando la idea del landshaft como un complejo natural-cultural, a partir de los años 30 esa visión se va sustituyendo por una noción privilegiadamente naturalista del paisaje. En Rusia predominó la vertiente naturalista, pues el propósito del país era dominar los grandes espacios, en un ambiente de centralización y autoritarismo, al contrario de Europa Occidental, donde lo fundamental fue la estructuración geopolítica, y el enraizamiento de la población en condiciones de dominio democrático. A partir de 1917, con la Revolución de Octubre, se reforzó la vertiente naturalista, se eliminó la concepción humanista y culturología, al pensarse que la sociedad era homogénea movida por la necesidad de construir el hombre soviético. La ciencia del paisaje sobre todo en la Universidad de Moscú desarrolló un complejo sistema de levantamiento cartográfico, operando en tres niveles taxonómicos: global, regional y local. Predominó la visión de la Geografía física en el estudio y análisis de los mismos.

La etapa racionalista naturalista de la primera mitad del siglo $\mathrm{XX}$ :

Se enmarcan en la influencia decisiva de los trabajos de Karl Troll, el cual propone los conceptos de Ecología del Paisaje y Geoecología. Este autor, les presta atención fundamental a las formaciones vegetales, apoyándose en una base naturalista, que es el concepto de paisajes. Más que todo la vegetación se considera como la fisionomía del paisaje.

El geógrafo alemán Troll (1966), por primera vez sugiere la creación de una nueva disciplina científica, designándola inicialmente con el término de "Ecología del Paisaje", en 1939, y después renombrándola en 1966, como "Geoecología". Troll definió a la Geoecología (primeramente, designada como Ecología del Paisaje) como "la ciencia sobre los complejos naturales, que condiciona las interrelaciones entre los seres vivos y su medio en la parte dada del paisaje". Ella estudia "los ecotopos", que son relativamente homogéneos de acuerdo a su contenido biólogo-ecológico, que son análogos, según su esencia, a las facies del paisaje, en la comprensión de Solntsev (1967). Troll define a la Geoecología sugiriendo un cambio terminológico para evitar las confusiones y “mejorar la comprensión de los científicos en los diferentes países", en tanto que a partir de los ecotopos se trata de sustituir a las "biogeocenosis" de B. N. Sukachev y usando el término de la Ecología del Paisaje se sustituye el término de Biogeocenología (Troll, 1950). Según Troll (1966) la Geoecología une en dos enfoques, el propio enfoque paisajístico, que estudia la diferenciación espacial de la superficie terrestre en interrelación entre los fenómenos naturales, y el enfoque biológico-ecológico, que investiga las interrelaciones funcionales de los fenómenos y complejos naturales como sistemas ecológicos. El científico estaba convencido, de que a la Geografía le era necesario conocimientos ecológicos profundos y la Ecología a su vez, deberá aún más que antes, darle la atención principal a la diferenciación espacial y a la cartografía de las asociaciones vivientes. De tal manera, de acuerdo a su idea de partida, es una disciplina paisajística con una dirección particular dirigida al conocimiento 
de las propiedades y funciones ecológicas de los paisajes (Leser, 1991).

Con el transcurso del tiempo, la Geoecología no solo se consolidó como la disciplina puente entre la Ecología y la Geografía, sino que amplió sus visiones y sus concepciones. En este sentido, fue fundamental el tener en cuenta no solo a los paisajes naturales y antroponaturales, sino también los culturales. De acuerdo a esta definición tradicional de Karl Sauer (1925), se incorporaba en el análisis los problemas relacionados con el paisaje visual, el perceptual y el valorizado y lo definió en el seno de la Ecología del Paisaje los productivos o económicos y los de gestión o manejo (Forman \& Godron, 1981; Naveh \& Lieberman, 1984).

Una definición muy clara y contemporánea de la Geoecología de esta etapa ha sido dada por Timashev, considerando que "es una rama de la Ciencia del Paisaje que estudia los complejos o paisajes naturales, antroponaturales y culturales (antropogénicos) de la Tierra, (las regiones, y los geosistemas de los continentes y los océanos) en los niveles global, regional y local, en calidad de medios de vida (de hábitat) de los organismos y los seres humanos, y de las actividades socio económicas de los grupos sociales (Timashev, 2007). Timashev (2008) definió como medio geoecológico, el entorno de la sociedad humana. La misma como disciplina geográfica, se considera una dirección particular dedicada al estudio del medio geográfico, a sus geosistemas, con el objetivo de solucionar los problemas relacionados con las actividades vitales de los seres humanos, como elemento de la naturaleza viva, y la obtención de los recursos necesarios para la existencia de la humanidad. El antropo y el eco centrismo, dirigido a estudiar los paisajes con vistas a la creación para los seres humanos y los organismos vivos de medios de hábitats favorables.
En su acepción de considerar al paisaje como un medio de hábitat, como un conjunto interrelacionado de formaciones naturales y antroponaturales la Geoecología asume al mismo como (Golubev, 2008):

Un sistema que contiene y reproduce recursos y servicios naturales.

Un medio de vida y de la actividad humana.

Una fuente de percepciones estéticas y de valores estéticos y culturales.

Un fondo genético.

Un laboratorio natural.

Sobre todo, a partir de 1980, el concepto de paisaje fue interpretado por los ecólogos provenientes de la Biología, para tratar de especializar a la noción biocéntrica de ecosistema. En este caso, se considera como el soporte para la existencia de fenómenos y recursos bióticos de diversos tipos. Se consideran así las matrices, las manchas, los núcleos, los nodos, los biocorredores, como elementos espaciales que se desarrollan en la superficie del planeta tierra. Se insiste, por lo tanto, más que en el complejo de los componentes naturales, en el soporte de los fenómenos bióticos de diverso tipo (Forman \& Godron, 1981). Se desarrolla la tendencia de a articulación entre la visión físico geográfica y la biologicista, a través del concepto de geosistema (Preobrazhenskii et al., 1982).

La etapa geosistémica de los años 1950-1980: A partir de la Segunda Guerra Mundial, debido a la incidencia de proyectos de intervención y de planificación para la reconstrucción de países devastados por la guerra, y la colonización y asimilación de inmensos espacios, se le presta una atención preferencial al entendimiento de la naturaleza como un todo. En la Unión Soviética, con el propósito de establecer los planes de asimilación de vastos espacios, y también en otros países del campo socialista 
(la República Democrática Alemana), se desarrolla toda la concepción del paisaje natural, trabajándose a tres niveles escalares: planetario, regional y local. Junto con el perfeccionamiento de los métodos de levantamiento de campo, con el auxilio de técnicas de fotointerpretación, se desarrolla la formulación de sistemas de unidades taxonómicas tanto de la tipología como de la regionalización (Solntsev, 1997).

A comienzos de los años 60, la idea de paisaje natural, es interpretada de acuerdo a los preceptos de la Teoría General de Sistemas, surgiendo así la teoría de los geosistemas naturales, elaborada en lo fundamental por V. B. Sochava, en la Siberia rusa a partir de comienzos de 1960 (Rodriguez, 2015). Siguiendo esa interpretación se desarrollan las direcciones del estudio de las propiedades sistémicas del paisaje natural (estructura, funcionamiento, dinámica, evolución, estabilización) (Figura 1). No obstante, continua la visión como un complejo natural espacial, o sea como el conjunto interrelacionado y totalizador de los componentes de la naturaleza.

Hay sin embargo opiniones de que esa visión de la Geografía soviética era exageradamente detallista y rígida, considerando al mismo como una máquina sustentada en una especie de Geografía molecular (Martínez de Pisón, 1999).

Otras nociones son desarrolladas posteriormente de manera paralela, tales como:

La concepción paisajística de los geógrafos franceses: Georges Bertrand desarrolló la idea de Geosistema/Territorio/Paisaje (GTP). En su visión, el paisaje es la expresión fisionómica visual y el geosistema es un orden de la clasificación jerárquica de los geosistemas naturales, en la que conjugaba en una misma interpretación los tipos y los individuos. Al territorio correspondía en lo fundamental los aspectos de la utilización y explotación económica, siendo lo más importante el análisis del potencial ecológico del paisaje apoyado en tres elementos: el potencial natural (formado por unidades o geosistémicas, en la que predominan los factores abióticos), la actividad biológica (factores bióticos). Es una interpretación biogeográfica de la realidad.

La Ecogeografía: desarrollada en lo fundamental por el geógrafo francés Jean Tricart, que insistió en el relieve, como elemento integrador de los suelos y la vegetación, estableciendo además una sistematización de la estabilidad del medio natural.

Los estudios integrados del paisaje, implementados por la escuela australiana, holandesa y de otros países, que se basaron en analizar las interrelaciones entre el relieve, los suelos y la vegetación. Algunos de los trabajos siguieron en lo fundamental la idea de las unidades locales establecidas por los especialistas soviéticos.

$\mathrm{Si}$ bien, en algunos momentos esas interpretaciones tomaban algunas ideas desarrolladas por la concepción naturalista/geosistémica, en general constituyeron interpretaciones específicas, que incluso se alejaron de las concepciones planteadas en la propuesta original de Sochava.

\section{La etapa cultural de 1990-2000:}

La Geoecología ha cobrado una fuerza inusitada a partir de finales del siglo XX. Estos nuevos tiempos exigen cada vez más del conocimiento totalizador, abarcador, relacional; precisan de una mirada amplia, multidimensional, holística. La temática ambiental se afianza en el quehacer práctico, y se convierte en una herramienta para programar el futuro. Para la ciencia en general, y para la Geografía en particular, es una oportunidad única, integrar todo el saber fragmentario, concentrar todo el conocimiento fragmentado, y erigirse como una potente fuerza en la sociedad del conocimiento que se va consolidando. El concepto fundamental de la Geoecología es el de paisaje, y su interpretación como geosistema. 
Desde una mirada holística en la ciencia contemporánea, se considera que el mismo está integrado por lo natural, el espacio productivo, lo cultural, y se manifiesta en lo estético y visual como una totalidad. Es la forma que adoptan los hechos geográficos, físicos y humanos sobre la superficie de la tierra. Igualmente, las representaciones que de ellos tenemos, los significados que se le otorga de modo personal o colectivo (Mata Olmo, 2014). Además, no consiste solo en la configuración material, en la fisionomía del territorio. Surge de la relación sensible, de la percepción sensorial (principalmente visual, aunque no solo) del territorio observado y vivido por el ser humano (González Bernáldez, 1981).

También se interpreta desde la perspectiva regional y territorial, como una cualidad específica de la región y del territorio que la apropia y gestiona. Se aplica a todo el territorio y se manifiesta en un paisaje que se percibe y se vive paisajísticamente.

En los paisajes "son individualizables las mutaciones sociales, la modificación de sus modos de producción, de las formas urbanas, de los modos de vida, de la actividad laboral y económica, sobre todo de la visión del mundo y de la vida" (Venturi Ferraiolo apud Mata Olmo, 2014, p. 57). El paisaje Distingue, identifica a un individuo geográfico, una especie de región geográfica, en la que se identifican un espacio natural y uno cultural a través de su morfología (Claval, 2010).

Se va consolidando, en algunos autores, la idea del paisaje como la expresión subjetiva por parte de los seres humanos del entorno, no solo natural sino fenoménico. Se desarrollan así los conceptos visuales, percibido y valorizado del paisaje. Muchas de estas nociones provienen de una interpretación particular en la Sociología y la Geografía, en la que en un término preferencial y desde posiciones humanistas, fenomenológicas y existencialistas, se coloca en primer lugar la idea como un fenómeno de la subjetividad. Es de destacar la emergencia de los conceptos de espacio y paisaje vivido, asociada a la internalización por parte de los individuos del entorno en que se desarrollan a su actividad vital y existencial. Se formulan así los conceptos de espacio o paisaje vivido e instituido (Farinelli, 2012).

Desde la Geografía Crítica el mismo se visualiza fundamentalmente, como la "apariencia" del espacio, representado en lo fundamental por la dimensión visual. En tales casos es muy común encontrar posiciones en que el paisaje natural de partida o sus modificaciones antropogénicas son dejadas a un lado, ocultadas o subvaloradas bajo la omnipresente existencia de la actividad humana (Kalutskov, 2009; OSE, 2009; Correa, 2012).

Se debe destacar el considerar del paisaje como la base para una de las formas de construcción de la llamada percepción geográfica. Martínez de Pisón (1999) es muy esclarecedor en este sentido cuando plantea que “... la lectura del paisaje es parecida a la lectura de un proceso cultural..." se habla del objeto como de un palimpsesto, es decir de escrituras superpuestas de un mismo documento. “...el paisaje del geógrafo es una imagen original de la Tierra...” (p. 415). “...la Geografía del paisaje es pues incisivamente una morfo geografía..." (p. 415). “...yo soy yo y mi paisaje, y si no lo salvo a él, no me salvo yo...” (p. 416).

El renacer de la Geografía Cultural, enfatiza en el análisis de las maneras en que los artefactos materiales son apropiados y sus significados transformados a través de hábitos sociales opuestos. Lo fundamental es estudiar las relaciones sociales y el significado simbólico revelado en acciones sociales en un determinado lugar o localidad. (Maia, 2001). La nueva Geografía cultural, considerada como el llamado "giro o renovación cultural" en la Geografía, tomando a Sauer como referencia, se 
diferencia en que consideran que los paisajes como objetos materiales no son neutros, sino que reflejan las relaciones de poder y las maneras dominantes de "ver" el mundo. Por lo tanto, los paisajes no son solo construidos, sino que también son percibidos a través de la representación de versiones ideales, en la pintura, en la poesía y también en el discurso científico y en los escritos académicos (Maia, 2001).

Denis E. Cosgrove, en los años 90, plantea la idea de la renovación o giro cultural de la Geografía Cultural. Considera al paisaje como un texto cultural. Propone tres maneras por las cuales la Geografía cultural moderna se mueve teóricamente: cultura y conciencia; cultura y naturaleza; cultura y poder. Para la realización de esa propuesta sugiere la lectura detallada del texto, que para los geógrafos quiere decir la lectura del paisaje en todas sus expresiones. Esta puede ser un recurso metodológico importante, porque a partir de esa lectura se podrán realizar profundos análisis del espacio geográfico representado, aunque sea momentáneamente, por ese paisaje. Se desarrolla así una "hermenéutica textual”, (Maia, 2001; Correa \& Rosendahl, 2003).

Para Cosgrove \& Jackson (2003), esta renovación se produjo a través de una cooperación entre la geografía cultural humanista y la geografía social marxista, en torno a la idea del paisaje como texto, que puede ser leído e interpretado como documento social. Según esta posición, el paisaje pasa a ser considerado como una imagen cultural, enfatizándose el papel de la simbología y considerándolo como una construcción cultural. De este modo, el concepto de paisaje se está considerando de manera hibrida en que se van conjugando los aspectos sociales y los perceptuales.

La etapa de hibridación del siglo XXI:

A partir de los cambios geopolíticos del sistema mundo a fines de los años 90 del siglo $\mathrm{XX}$, la ciencia está derivando hacia una visión post clásica, reflejo de una nueva revolución científica global (Krasovskaia, 2010; Krasovskaia et al., 2016; Dirin, 2018). La esencia de esta etapa consiste en que se colocan en primer plano las formas interdisciplinarias y orientadas problémicamente de la actividad científico-investigativa. Se debilita la influencia de los factores interdisciplinarios en el desarrollo de la ciencia, pero se acrecienta la presencia de los "hábitos paradigmáticos" y de las ideas que provienen de otras ciencias. La Geoecología post clásica se encamina a la investigación de los sistemas correlativos (correlacionales) de nuevo tipo, cuyas propiedades adaptativas se forman no solo por los procesos naturales, sino también por procesos sociales, económicos y políticos. Actúan aquí las relaciones correlacionales o de adaptación entre los componentes ya creados por otros sistemas. A diferencia de la utilización de la naturaleza geográfica, que analiza los sistemas correlacionales integrales pares, tales como medio natural-socium, medio natural-economía, etc., la nueva geoecologia post clásica no se ocupa de los múltiples sistemas correlacionales integrales, sino de los bloques de los sistemas socio naturales que se manifiestan en el proceso de la utilización de la naturaleza (Figura 2).

De tal manera, tres son los nuevos aportes de esta Geoecología Post Clásica:

La formulación teórica de la noción esencialmente geográfica, de sistema socio natural (Krasovskaia, 2010; Krasovskaia et al., 2016), o sistema geoecológico del paisaje (Romanova et al., 2010), se diferencia del de socio eco sistema desarrollado por Maas et al. (2003) y Sarukhán (2007) con una visión ecólogo-biologizante en el marco del proyecto de las Naciones Unidas sobre "Ecosistemas del Milenio." 


\section{SISTEMA SOCIO NATURAL Y LAS INTERACCIONES FUNCIONALES EN EL MISMO (T.M.KRASOVSKAIA, 2008,2016)}

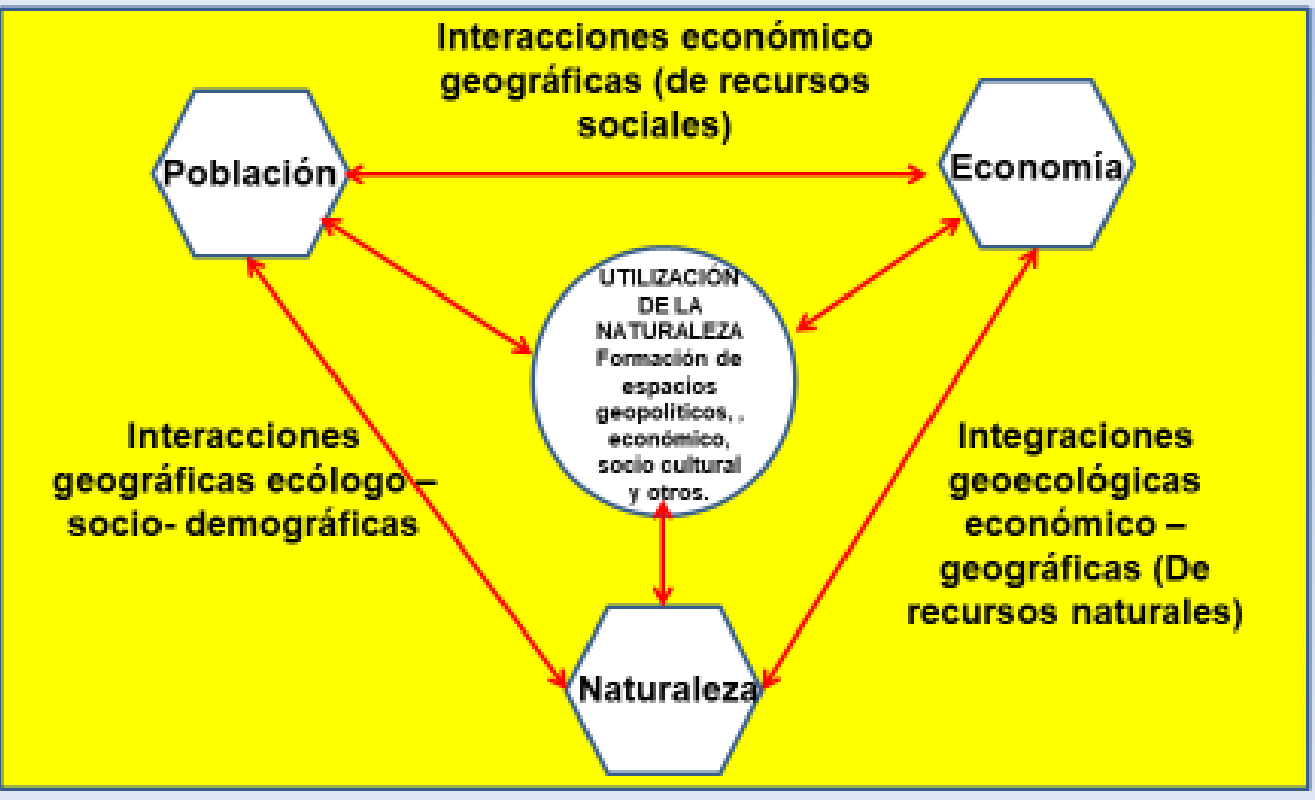

FIGURA 2 - Sistema socio - natural y las relaciones funcionales en el mismo.

FUENTE: Krasovskaia (2010); Krasovskaia et al. (2016).

Para la investigación de los sistemas socio naturales (SSN) complejos, con ayuda del enfoque sistémico, lo más efectivo se logra en la combinación y utilización de los medios contemporáneos de la Geoinformática y la Geosinergética (Karpujin, 2010). La Geosinergética, como dirección interdisciplinaria, es la teoría de los sistemas auto organizados que analiza las relaciones entre los elementos de la estructura (subsistemas) que se forman en sistemas abiertos (geográficos, biológicos, geológicos). Se trata de procurar salidas prácticas, que se manifiestan en los procesos de planificación y gestión de paisajes, incorporando a la innovación geoecológica, como el mecanismo preferencias de la relación medio ambiente-tecnología.
El desarrollo de la Geoecología del período post clásico estimula la solución de las demandas prácticas y forma un importante fundamento científico para el análisis de la diversidad de los sistemas correlacionales con el propósito de asegurar el tránsito hacia el desarrollo sostenible. El enfoque sinérgico que es la base de tal análisis permite integrar muchos procedimientos metódicos de las ciencias naturales y las humanitarias (Krasovskaia, 2010; Krasovskaia et al., 2016).

De tal manera, la Geoecología de los Paisajes, se está refirmando cada vez más como la concepción idónea para encarar de manera inter y transdisciplinaria la cuestión ambiental. La Geoecología tiene de tal manera las siguientes ventajas como base ob- 
jetiva para operacionalizar el paradigma ambiental (Golubev, 2008).

Parte de distinguir, delimitar, caracterizar y cartografiar las unidades del espacio o paisaje natural, que son la expresión objetivamente existente del orden natural sobre la superficie del planeta Tierra.

A partir de esas unidades naturales, como denominador común se pesquisa la forma en que las mismas han sido moldeadas y transformadas por la acción humana, desde dos perspectivas: desde una visión "objetiva" de su transformación en formas de uso, partiendo de la explotación del potencial de recursos y servicios del que son portadores. Esa visión objetiva permite distinguir paisajes de acuerdo a un tipo de uso, que establece un patrón de utilización, y la presencia de objetos que responden a ese uso principal, todo lo cual se refiere en una morfología.

Desde una visión subjetiva, a través de las nociones de paisaje antropo natural (análogo al de espacio geográfico), se permite visualizar la forma en que los seres humanos ocupan, asimilan e incluso se apropian de la superficie del globo terráqueo, y modelan las actividades socio económicas y culturales transformando los paisajes naturales, y utilizando de acuerdo a determinadas condicionantes históricas, políticas y económicas el medio natural.

La Geoecología constituye una base certera para interpretar el "medio exterior", brinda algunas "pistas" para comprender como es el medio interior de los seres humanos. Así desde una perspectiva más subjetiva de la forma en que los seres humanos perciben y valoran la naturaleza, los espacios y los paisajes.

Justamente la temática de la percepción del paisaje permite entender como la gente visualiza e incluso valoriza su entorno, sobre todo desde la visión simbólica y cultural. La noción de paisaje cultural permite considerar la forma en que la naturaleza y sus modificaciones son apropiadas y construidas culturalmente. Por otra parte, constituye un verdadero enlace o eslabón interactuante con el medio personal, íntimo de los seres humanos, o sea con su naturaleza interna.

Si a ello se suma la posibilidad de añadir una perspectiva proveniente de la Biología, a través de la Ecología del Paisaje, que se fundamenta en la interpretación biótica del entorno, es posible articular diáfanamente la dimensión de la espacialidad y la territorialidad que brinda la Geografía a la dimensión más funcionalista y verticalista que ofrece la Bioecología.

Desde el punto de vista evolutivo la visión paisajística permite entender la forma en que los paisajes naturales han sido humanizados, dando lugar al cambio de la composición y estructura de su composición como formación natural, y a su modelamiento y como base para la actividad productiva y social de la sociedad humana y a su conformación como lazo con la subjetividad humana, tanto bajo la forma de imagen, así como mediante la estructuración de elementos de su identidad regional y territorial.

\section{Innovación y Geoecología}

¿Cómo puede colaborar la Geoecología de los Paisajes a tratar de construir una dimensión tecnológica sustentable del medio ambiente? En el último cuarto del siglo XX ha comenzado una nueva revolución científica global, que se manifiesta por medio del surgimiento de la etapa post clásica de la ciencia. La esencia de esta etapa consiste en que se colocan en primer plano las formas interdisciplinarias y orientadas problémicamente de la actividad científico-investigativa. Se debilita la influencia de los factores interdisciplinarios en el desarrollo de la ciencia, pero se acrecienta la presencia de 
los "hábitos paradigmáticos" y de las ideas que provienen de otras ciencias. A fines del siglo XX el hombre se posiciona no ya como el dominador del mundo, no como el creador de sus leyes, sino como el copartícipe del proceso mundial. El imperativo del desarrollo de la ciencia post clásica, ha determinado el desarrollo de la geoecologia contemporánea geográfica, que estimula la necesidad de la solución de muchas tareas prácticas para el tránsito hacia el desarrollo sostenible. La Geoecología post clásica se encamina a la investigación de los sistemas correlativos (correlacionales) de nuevo tipo, cuyas propiedades adaptativas se forman no solo por los procesos naturales, sino también por procesos sociales, económicos y políticos.

En la actualidad, la ciencia y la tecnología se introducen cada vez más en la práctica social a través de la innovación (Drozdov, 2010). La innovación es ya una introducción de algo nuevo, que posee una alta efectividad o son inversiones en trabajos perspectivos para la realización y ejecución de novedades, la introducción de medios para elaborar nuevas técnicas, tecnologías e investigaciones científicas especiales. Para que haya innovación, se necesita producción del conocimiento nuevo pertinente, específico, concreto. Ello se conoce como novedad, o novación y nuestro objetivo es convertir las novaciones en innovaciones. ¿Cómo se logra eso?, la Geografía de la Innovación constituye un esquema principal que genera procesos de innovación. Hay dos caminos claves:

- Incorporar en el análisis geoecológico (paisajístico espacial) los avances tecnológicos, las nuevas tecnológicas, los conocimientos de otras disciplinas, los conceptos y categorías de interfase. Todo ello para adaptarlo a la espacialidad, a la territorialidad concreta de los sistemas geográficos.

- El otro camino es la utilización de la propia Geografía en la solución de nuevas tareas y el estudio de nuevos territorios. La búsqueda de procedimientos metódicos para la solución de tareas concretas, o de modos de análisis de datos experimentales en el arsenal de las disciplinas de la propia Geografía. Estos resultados de los procedimientos standards se enriquecen y se transforman de manera significativa. Un ejemplo clásico de tal tipo es la formación de los Sistemas de Información Geográfica.

En el amplio espectro de temas y esquemas de los cuales se fundamenta la innovación geoecologia espacial (geográfica) es posible establecer la siguiente lista de presencia:

Primer grupo: Geoinformática, tecnología de computación y Sistemas de Información Geográfica.

Segundo grupo: educación, principalmente escolar, formación universitaria.

Tercer grupo: planificación y gestión del paisaje para la conformación del medio ambiente, evaluación de los impactos, estudios de vulnerabilidad y riesgos.

Cuarto grupo: energía alternativa, recursos y condiciones naturales, servicios ecosistémicos.

Quinto grupo: elaboración de los problemas teóricos y metodológicos de la Geografía.

La Geografía y en particular la Geoecología, tienen mucho que aportar en la incorporación de la dimensión tecnológica a la construcción del medio ambiente, aunque en realidad en ocasiones o no lo ha hecho, o no lo ha valorado, o no se ha divulgado y reconocido. Los geógrafos no pueden esperar a que las demandas caigan por si solas. Tienen que ser proactivos, creadores, en una palabra, innovadores.

\section{Consideraciones finales}

Es obvio que no es fácil romper con el facilismo, el reduccionismo y la excesiva simplificación que se ha entronizado en la visión popular, mediá- 
tica y en los estudios técnicos banalizadores (sobre todo vinculados a los Estudios y Evaluación de Impacto Ambiental). Esta ha sido una dimensión tecnológica ineficaz que por desgracia aun predomina en muchos medios.

Sin embargo, en el ambiente de mayor rigor, seriedad y complejidad que se va construyendo a partir de 1992 con la celebración de la Cumbre sobre Medio Ambiente y Desarrollo celebrada en Rio de Janeiro, Brasil, la cuestión ambiental exige de conocimiento fundamentado, de rigor teórico metodológico, de datos empíricos colectados sobre la base de fundamentos serios. Justamente esa visión es ofrecida por la Geoecología, que en dos siglos de existencia ha logrado construir y establecerse como un enfoque objetivo y científicamente fundamentado capaz de ser la base sobre la cual se articule todo el conocimiento disciplinario inherente al paradigma ambiental.

La Geoecología como propuesta metodológica se sustenta en un sistema filosófico coherente caracterizado por (Moraes, 1994; Rodriguez, 2015): una ontología, que caracteriza con claridad al paisaje como sistema de conceptos como su objeto de análisis; una gnoseología, que opera en las diferentes categorías de considerar al paisaje como un espacio natural, un espacio antroponatural que asimila e in-

\section{Referencias}

Baklanov, P. I. A. Estructuración del espacio geográfico: fundamento de la geografía teorética. In: Artiboletskii, S. S. et al. La Teoría de la Geografía socio-económica. Estado actual y perspectivas de desarrollo. Conferencia científica Internacional. Rostov na Donu, mayo, pp.12-21, 2010. Editorial de la Universidad Federal del Sur, 2010.

Bellón, M. R. La búsqueda del desarrollo sostenible: el papel del conocimiento de indígenas y campesinos. Revista Latinoamericana de Economía, 23(91), 37-45, 1997. terpreta las nociones de espacio geográfico y espacio social - y un espacio cultural, que incluye las nociones de visual, perceptual y valorizado; una lógica, que permite articular los elementos y componentes que entran en los diferentes niveles estructurales del enfoque; una epistemología, que permite la construcción del conocimiento utilizando un sistema de métodos, principios, técnicas y procedimientos adecuados al objeto y las categorías de estudio.

Es hora de ir a la articulación entre las disciplinas para reforzar el paradigma ambiental, para concentrar los esfuerzos, para ofrecer soluciones y salidas a los embates que provienen del negativismo, del reduccionismo economicista, del oportunismo académico, se necesita diálogo y no la estéril confrontación. La ciencia tiene que alzarse por encima de las mezquindades para mostrar que la sociedad del conocimiento es una imperiosa exigencia para vencer el caos, la incertidumbre y la apatía en la que está sumida parte de la humanidad en el siglo XXI. La Geoecología, armada de una potente base metodológica, con una experiencia de más de dos siglos de resultados prácticos y con una vocación para el dialogo inter y transdisciplinario se erige cada vez más como el núcleo fundamental en la construcción contemporánea del paradigma ambiental.

Benton, T. W. et al. What an Earth is to be done. A RedGreen Dialogue. Red Green Srtudy Group, Manchester, 1995.

Claval, P. Terra dos Homens: a Geografia. São Paulo: Editora Contexto, 2010.

Correa, R. L. Espaço e simbolismo. In: Castro, I. E. de et al. Olhares geográficos: modos de ver e viver o espaço. Rio de Janeiro: Bertand Brasil, 2012. p.133-153. 
Correa, R. L.; Rosendahl, Z. Introdução a geografia cultural. Rio de Janeiro, Bertrand Brasil, 2003.

Cosgrove, D. E.; Jackson, P. Novos rumos da Geografia Cultural. In: Correa, R. L.; Rosedahl, Z. Introdução a Geografia Cultural. Rio de Janeiro: Bertrand Brasil, 2003, pp. $135-146$.

Diakonov, K. G. et al. La ciencia del paisaje y los desafíos del tiempo (en ruso) In: Kotliakov, V. M. et al. Horizontes de la Ciencias del Paisaje, Problemas de la Geografia. Colección 138, Moscú, Casa Editorial “Kodeks”, 2014, pp.13-25.

Dirin, A. A. El concepto de espacio geocultural en la Geografía Social (en ruso). Revista ARGOS, 7, 146-154, 2018.

Drozdov, A. N. El espacio de las innovaciones geoecológicas, pp. 19-23. In: Romanova, E. P. (Redactor principal). La innovación en geoecologia: teoría, práctica, educación. Materiales de la conferencia de toda Rusia, Moscú, Facultad de Geografía, UEM, 2010.

Farinelli, F. La nature du paysage. Proceedings of the Council of Europe Celebrations on the $10^{\text {th }}$ anniversary of the European Landscape Convention, Strasbourg, Council of Europe, 2012. p. 86-88.

Forman, T. T.; Godron, M. Patches and structural components for a Landscape Ecology. BioScience, 31(10), 733-740, 1981.

Fussler, C. The cure for marketing myopia: meeting emerging market needs for eco - efficient products. Industry and Environment. 18(4), 34-37, 1995.

Gallopin, G. El medio ambiente humano. In: Estilos de desarrollo y Medio Ambiente en América Latina. México D.F.: Fondo de Cultura Económica, 1980. p. 205-235.

Golubev, G. N. La crisis ecológica global y la salvación de la humanidad (en ruso). In: Alekseev, B. A. (Org.). El mundo de la Geoecología (en ruso). Moscú: GEOS, 2008. p. 5-11.

González Bernáldez, F. Ecología y Paisaje. Madrid: H. Blume Ediciones, 1981.

Kalutskov, V. N. El landshaft en la Geografia cultural (en ruso). Moscú: Facultad de Geografía de la Universidad Estatal de Moscú, 2008.

Kalutskov, V. N. Problemas de la representación del Paisaje Cultural (en ruso). Vestnik: MGU, No.5, p. 22- 27, 2009.
Karpujin, S. S. La Geosinergética: dirección científica innovativa interdisciplinaria de la Geoecologia (en ruso), pp.2629. In: Romanova, E. P. (Redactor Principal). La innovación en Geoecología: teoría, práctica, educación. Materiales de la Conferencia de Toda Rusia. Moscú: Facultad de Geografía de la UEM, octubre del 2010.

Krasovskaia, T. M. Sistemas correlaciónales en la geoecologia post clásica. In: Romanova, E. P. (Org.). La innovación en la Geoecología: teoría, práctica, educación. Materiales de la conferencia de toda Rusia. Moscú: Facultad de Geografía, UEM, 2010. p. 30-33

Krasovskaia, T. M. et al. Introducción a la utilización de la naturaleza (en ruso). Moscú: Facultad de Geografía, UEM, 2016.

Leff, E. Ecología y Capital: racionalidad ambiental, democracia participativa y desarrollo sustentable. México DF: Siglo XXI Editores, 1994.

Leser, H. Ecología del Paisaje, Enfoques, modelos, metódicas, aplicaciones (en alemán). Stuttgart: Verlag Eugen Ulmer, 1991.

Maas, J. M. et al. Manejo del agua en la Cuenca del Río Cuixmala: bases ecológicas y sociales para un manejo integrado de cuencas. Proyecto Financiado por PAPIIT, UNAM. 2003.

Maia, D. S. A Geografia e o estudo dos costumes e das tradições. Terra Livre, São Paulo, n. 16, pp. 71-96, 2001.

Martínez de Pisón, E. La dinámica natural del Paisaje. In: Anais El territorio y su imagen. XVI Congreso de Geógrafos Españoles, Ponencias y Mesas Redondas. Centro de Ediciones de la Diputación Provincial de Málaga, España, p. 9-26, 1999.

Mata Olmo, R. Conocimiento geográfico del paisaje y políticas públicas. Estudios y experiencias de gestión a distintas escalas. In: Checa-Artasu, M. M. et al. Paisaje y territorio. México D.F: Universidad Autónoma Metropolitana, Tirant Humanidades, 2014. p. 49-88

Moraes, A. C. R. Meio ambiente e ciências humanas. Editora Hucitec; São Paulo, 1994.

Naveh, Z.; Lieberman, Y. S. Landscape ecology: theory and application. Sprienger New York: Verlag, 1984. 
Nikolaiev, V. A. Ciencia del Paisaje. Seminarios y Tareas prácticas. (en ruso), Facultad de Geografía de la UEM, 2006.

Ortega y Gasset, J. Obras completas. Volumen I. Madrid: Taurus/ Fundación José Ortega y Gasset, 2004, 757 p.

Preobrazhenskii, V. S. et al. Protección de los paisajes. Diccionario interpretativo. (en ruso). Moscú: Editorial "Progress", 1982.

Rodriguez, J. M. M. Teoría y Metodología de la Geografía. La Habana: Editorial Universitaria Félix Varela, 2015.

Rodriguez, J. M. M.; Suarez, C. La ciencia y la tecnología en el debate ambiental. Filosofía y Sociedad, T. II. La Habana: Editorial Félix Varela, p. 720-741., 2000.

Rodriguez, J. M. M.; et al. Geoecologia das Paisagens: uma visão geossistémica da analise ambiental. Fortaleza: EDUFC, 2004.

Rodriguez, J. M. M.; Da Silva, E. Para una interpretação epistemológica de la Geografia a partir de la Dialéctica. Mercator, 4(9), 55-68, 2006.

Romanova, E. P. et al. Evaluación Geo ecológica de los Paisajes (en el ejemplo del territorio de Netherlands). Vestnik $M G U$, Serie 5, 1, Geografía, 3-10, 2010.

Santos, M. Metamorfoses do espaço habitado. São Paulo: Hucitec, 1994.

Santos, M. A natureza do espaço: técnica e tempo, razão e emoção. São Paulo: Hucitec, 1996.

Santos, M.; Silveira, M. L. O Brasil: território e sociedade no início do século XXI. $2^{\mathrm{a}}$ ed. Rio de Janeiro: Editora Record, 2001.

Sarukhán, J. et al. Desarrollo interdisciplinario de modelos conceptuales y herramientas metodológicas para el estudio de los servicios ecosistémicos. Proyecto de Investigación SEP CONACYT 50955. México DF: IE/UNAM, 2007.

Sauer, C. O. Hacia una Geografia histórica: Discurso a la Asociación Norteamericana de Geógrafos, Baton Rouge,
Lousiana, diciembre de 1940. GEOCALL, "El pensamiento geográfico de Carl O. Sauer, año 10, n. 20, pp.13-67, 2009.

Sauer, C. O. The morphology of landscape. University of California, Publications I Geography, 2(2), 19-54, 1925.

Solntsev, V. N. Concepción poli estructural del agro paisaje. (en ruso), In: Gennadiev, A. N. et al. (Orgs.). Cambios del medio natural: aspectos globales y regionales. Moscú: Editorial de la Universidad Estatal de Moscú, 1997. p.17-29.

Solntsev, V. N. ¿En qué se diferencian las facies y las biogeocenosis? (en ruso). Revista de la Universidad Estatal de Moscú, Serie Geografía, 2, 184-187, 1967.

Sosa, N. M. Perspectiva ética. Madrid: UNED, 1995. (Serie Monografías)

Thayer, R. L. Gray world, green heart, technology, nature and the sustainable landscape. New York: Josh Wiley Sons Inc.,1994.

Timashev, I. E. Diccionario geoecológico interpretativo ruso - ingles (en ruso), Moscú: Casa Editorial $\mathrm{Mu}-$ ravei-Guide, 1999.

Timashev, I. E. El componente geoecológico principal del paisaje terrestre. In: Alekseev, B. A. (Org.). El mundo de la Geoecología (en ruso). Moscú: GEOS, 2008. pp. 11-20.

Timashev, I. E. La Geoecologia como ciencia ecólogo paisajística (en ruso). In: Vestnik MGU, n. 1, pp. 5-11, 2007.

Troll, C. El paisaje geográfico (en alemán). Hamburg: Studium Generale, 1950.

Troll, C. Landscape Ecology. Delft: ITC UNESCO Centre. 1966. (Especial Publication, s. 4)

Wulf, A. A invenção da natureza: a vida e as descobertas de Alexander Von Humboldt. São Paulo: Editorial Planeta, 2016.

Zonnelveld, I. S. Land ecology: an introduction to landscape ecology as a base for land evaluation, land management and conservation. Amsterdan: Academic Publishing, 1995. 